\title{
PALAEONTOLOGY
}

\section{Erratum to: Phylogenetic relationships of psammosteid heterostracans (Pteraspidiformes), Devonian jawless vertebrates by Vadim Glinskiy}

UUID: http://zoobank.org/B562A2FB-8B57-47DC-91D1-755CC784DD31

\begin{tabular}{|l|l|l|l|l|}
\hline Page & Column & Line (from top) & \multicolumn{1}{|c|}{ For } & \multicolumn{1}{c|}{ Read } \\
\hline 233 & right & $\begin{array}{l}47, \\
\text { heading }\end{array}$ & $\begin{array}{l}\text { Psammosteidae family } \\
\text { incertae sedis }\end{array}$ & $\begin{array}{l}\text { Psammosteoidei family incertae } \\
\text { sedis }\end{array}$ \\
\hline 235 & right & 18 & Psammosteidae & Psammosteinae \\
\hline 243 & right & 62 & (1) compact & (1) trabecular (spongy appearance) \\
\hline
\end{tabular}

The original article can be found online at https://biocomm.spbu.ru/article/view/648 https://doi.org/10.21638/11701/spbu03.2017.402

Citation: Glinskiy, V. 2018. Erratum to: Phylogenetic relationships of psammosteid heterostracans (Pteraspidiformes), Devonian jawless vertebrates. Bio. Comm. 63(3): 202. https://doi.org/10.21638/ spbu03.2018.306

Author's information: Vadim Glinsky, Researcher, orcid.org/0000-0001-9151-1161 Manuscript Editor: Nikita Zelenkov, Borissiak Paleontological Institute, Russian Academy of Sciences, Russia

Copyright: @ 2018 Glinskiy. This is an open-access article distributed under the terms of the License Agreement with Saint Petersburg State University, which permits to the authors an unrestricted distribution and self-archiving free of charge. 